\title{
Strain measurements with fibre Bragg grating sensors under inhomogeneous deformations
}

\author{
Susann Hannusch ${ }^{1)}$, Edgar Peretzki ${ }^{11}$, Katharina Schich ${ }^{1)}$, Thomas Lehmannn', Jörn Ihlemann ${ }^{1)}$ \\ 1) Professorship of Solid Mechanics, susann.hannusch@mb.tu-chemnitz.de, \\ edgar.peretzki@mb.tu-chemnitz.de, katharina.schich@mb.tu-chemnitz.de, \\ thomas.lehmann@mb.tu-chemnitz.de, joern.ihlemann@mb.tu-chemnitz.de, \\ Chemnitz University of Technology, Reichenhainer Straße 70, 09126 Chemnitz, Germany
}

\section{Keywords}

Fibre Bragg Gratings, FBG Sensors, Inhomogeneous Deformations, Strain Measurement

\begin{abstract}
Bragg grating sensors are fibre optic sensors for strain and temperature investigations with many advantages: the sensors can be embedded in plastic materials or composites and several gratings can be inscribed in one sensor. However, inhomogeneous deformation or transversal loading cause widening and splitting of the reflected wavelength peak of a fibre Bragg grating (FBG) sensor. These effects are shown in a residual stress analysis, in which the hole drilling method is adapted for FBG sensors. Additionally, a four-point bending test on three different notched aluminium beams is used to investigate the widening and splitting of the reflected peaks and their effects on the strain analysis. At each sample, a reference strain gauge sensor and two FBG sensors are applied. The two FBG sensors are loaded with different strain gradients. The unnotched beam and the beam with small strain gradient show the accuracy and reproducibility of the experiment. The beam with medium strain gradient shows no peak splitting, but the widening does influence the strain analysis. The results of the beam with high strain gradient demonstrate the peak splitting and the failure of the strain analysis methods. Initial approaches on how to deal with this widening and splitting are discussed.
\end{abstract}

\section{Introduction}

\subsection{Basic challenges}

The synergetic combination of different materials is an advantageous measure for the design and development of modern lightweight structures. Therefore, efficient lightweight construction solutions offer great potential for use in many technical applications. Within the Federal Cluster of Excellence MERGE, the interdisciplinary research area IRD F deals with advanced scientific simulations as well as the optimisation and dimensioning of multifunctional lightweight structures [1-3]. Besides the theoretical investigations, experimental analyses are required for material characterisation and validation of the simulations.

During manufacturing of polymer-based lightweight structures, significant residual stresses can occur (e.g. due to shrinking processes). Hence, the experimental analysis of residual stress formation and the investigation of existing residual stress states are particularly important topics of the research activities. A well-known and proven method for residual stress analyses is the hole drilling method, which is commonly based on strain detection by strain gauges at the specimen surface [4]. To determine residual stress states at different depths of the specimen, a new approach has been developed. The method enables strain measurement at the place of occurrence of the residual stresses, i.e. inside the structure. For this purpose, fibre Bragg grating sensors were integrated into an epoxy-based polymer material. 
Caused by inhomogeneous deformations (e.g. resulting from notch effect) or transversal stressing of the fibres, a widened the FBG spectrum and splitting of narrowband peaks can occur [5], which makes standard strain evaluation inapplicable. Due to the observation of this phenomenon during the performed residual stress analyses, further fundamental tests were carried out, which enable a better understanding of the occurring strain distribution and the resulting spectrum of the FBG sensors. These tests include measurements with FBG sensors which are applied to the sample surface with a defined characteristic strain profile at the measurement section.

\subsection{Fundamentals of fibre Bragg grating sensors}

Fibre Bragg grating sensors are fibre optic sensors with inscribed fibre Bragg gratings, which are described in [6], among others. The structure and a schematic principle of a fibre Bragg grating as well as a small application example are depicted in Figure 1. The core and the cladding consist of silica glass with different refractive indices, which enables a total reflection. This total reflection is required to transport the light into the core. The coating is a mechanical protection and consists of plastic, ceramic, or other materials. The sensors used are Draw Tower Gratings and they are coated with ORMOCER $\AA$, a special mixture of plastic and ceramic [7]. The injected light spectrum is generated by a tunable laser, as shown in Figure 1 (top, left). The interrogator system used is the FBGscan 800 from FBGS Technologies. The inscribed Bragg grating is a change of the refractive index of the core, which produces a reflection of the injected light. The reflected spectrum is called Bragg wavelength $\lambda_{B}$ and depends on the effective change of the refractive index $n_{\text {eff }}$ and the grating period length $\Lambda$. The Bragg wavelength can be calculated by:

$$
\lambda_{B}=2 \cdot n_{\text {eff }} \cdot \Lambda
$$

When the fibre is heated or strained in the region of the Bragg grating, the effective refractive index $n_{\text {eff }}$ and the grating period length $\Lambda$ change. This variation is detectable by a shift in the wavelength peak, as shown in the centred example at the bottom of Figure 1. As a result of the shift of the reflected wavelength, the thermal and/or mechanical strain is calculated by:

$$
\frac{\lambda_{B}-\lambda_{B, 0}}{\lambda_{B, 0}}=k \cdot \varepsilon+\propto \cdot \Delta T,
$$

where $\lambda_{B, 0}$ is the Bragg wavelength at the beginning, $k$ is the $\mathrm{k}$-factor or photoelastic coefficient, and $\alpha$ represents the sensitivity to temperature changes. Moreover, the symbol $\varepsilon$ represents the mechanical strain along the fibre axis and $\Delta T$ is the temperature change. An assumption for Equation (2) is that the peak of the reflected wavelength is narrowband [6]. 


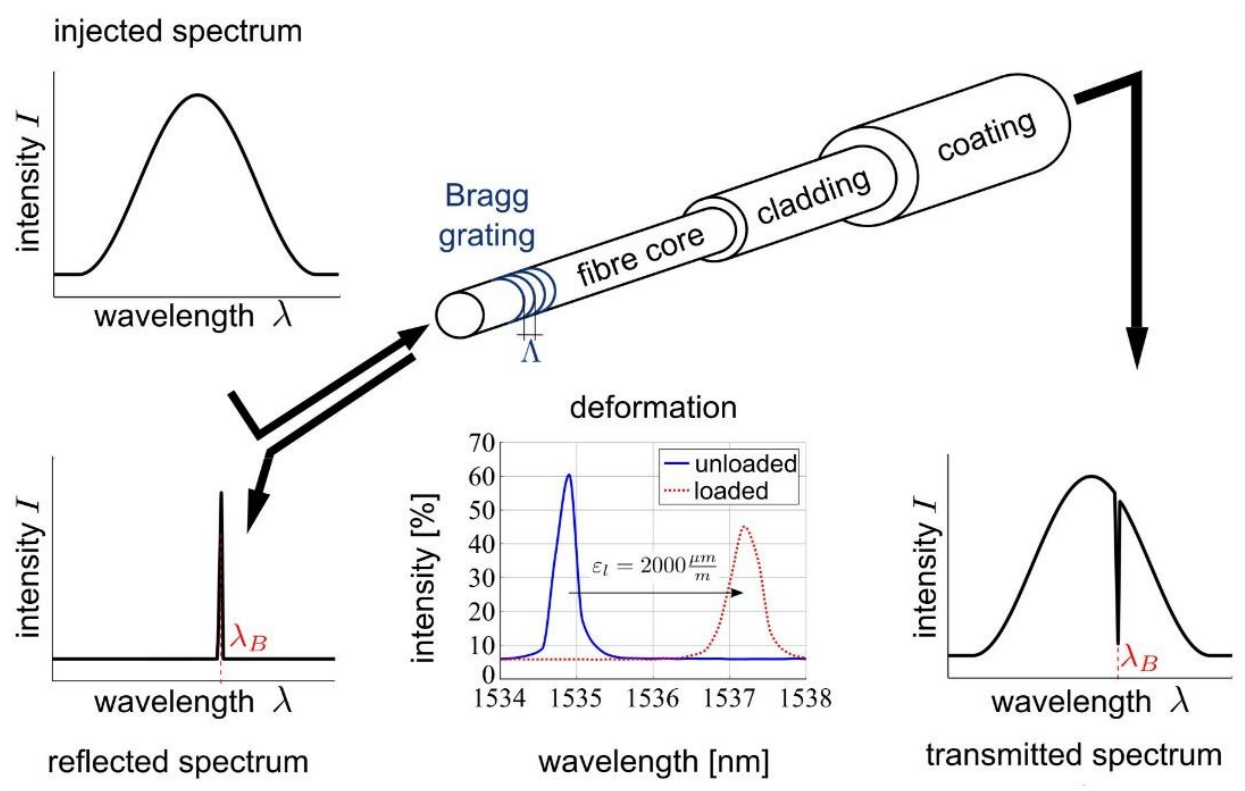

Figure 1: Structure and schematic principle of a fibre Bragg grating and a small application example

\section{Residual stress analysis with fibre Bragg grating sensors}

\subsection{Motivation}

A well-known method for the analysis of residual stresses is the hole drilling method in combination with an electrical strain gauge rosette [4]. The strain gauge rosette is applied to the surface of a sample. Drilling a hole in the centre of the rosette causes deformations because the residual stresses are rearranged. The strains are detected by the strain gauges. The residual stresses can be calculated with the help of different evaluation methods such as the integral method or MPA method after Kockelmann and Schwarz. However, there is a major disadvantage in the use of these methods. It is only possible to investigate the residual stresses near the surface as a function of the hole diameter. Residual stresses in several millimetres of depth cannot be determined. In the following section, the method described above is adapted using FBG sensors for the residual stress analysis inside a component.

\subsection{Experimental setup}

For the investigation of residual stresses, first, a nearly stress-free specimen was manufactured, which contains an electrical strain gauge rosette for the hole drilling method and a FBG sensor with nine inscribed FBGs to analyse the residual stresses at different depths, see Figure 2. In order to obtain a known stress state in the sample, steel plates are adhered to the sample, which serve to induce a homogeneous pressure in the sample by means of a force.

The strain gauge rosette is used to determine the residual stresses on the surface of the sample as a reference measurement method. The stress states at three different depths are analysed using three FBG arrays with three FBGs each. They are embedded with epoxy resin between unreinforced epoxy plates. This results in a sample consisting of one material (epoxy) with integrated sensors. The positions of the FBGs correspond to the strain gauges. The strain gauges measure radial and the FBGs tangential strains. This approach uses the advantages of the FBGs such as the thin structure, chemical stability, high precision, and multiplexing, which allows the use of several measuring gratings in one fibre. The height of the sample is $10 \mathrm{~mm}$.

The residual stresses around the FBGs are also rearranged by drilling a hole. The process of the residual stress analysis is carried out as follows. First, the homogeneous stress state is applied by the external compression force. Then a 2-blade cutter with a diameter of $5 \mathrm{~mm}$ is used to drill the first part of the hole with a depth of $1 \mathrm{~mm}$. Because the temperature increases during drilling, a delay time of 30 minutes is taken into account before the next $1 \mathrm{~mm}$ step is drilled. This process is repeated until the hole is drilled through the epoxy plates. 


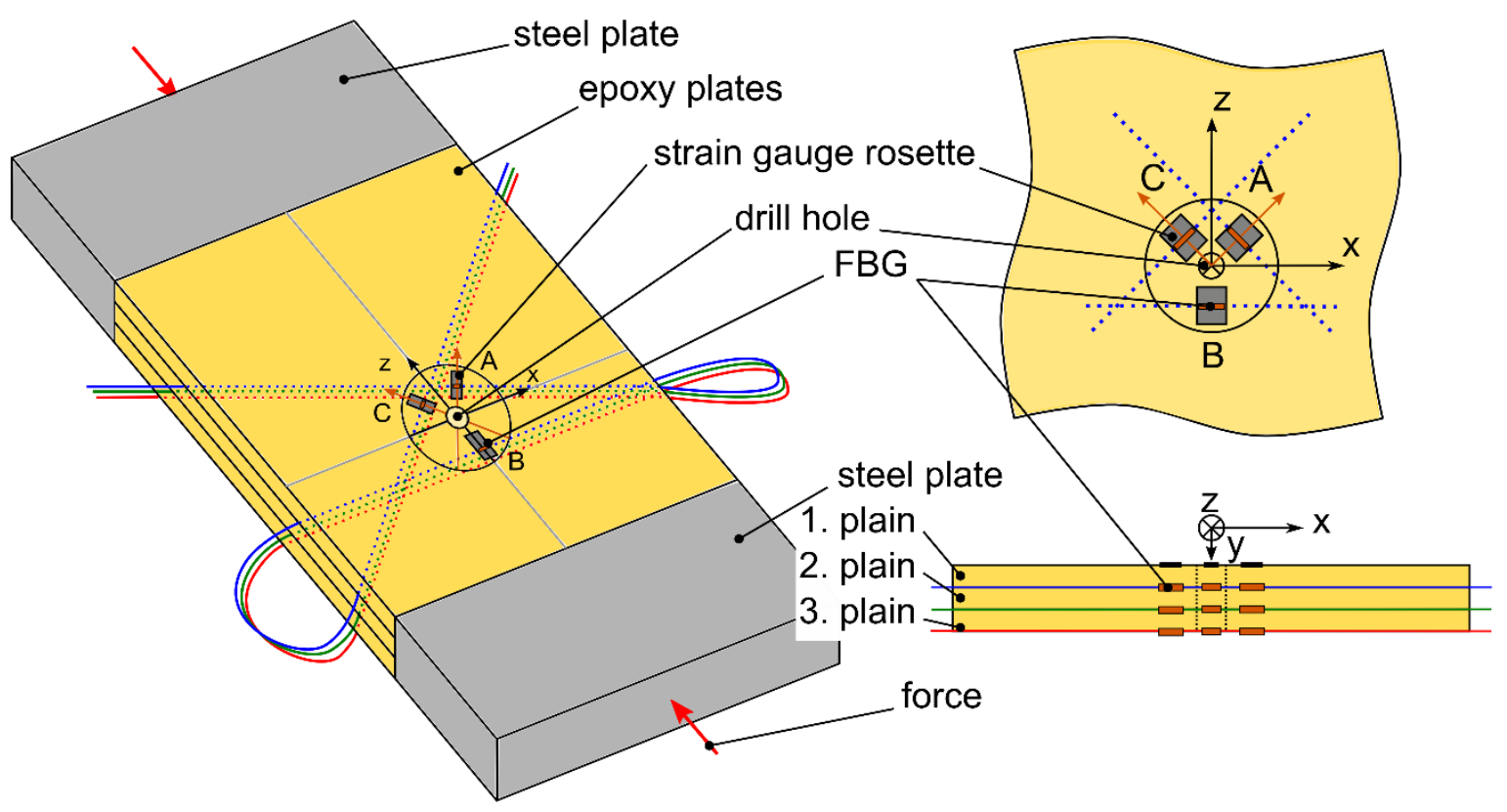

Figure 2: Experimental setup for the hole drilling method with epoxy sample

\subsection{Results and challenges}

Figure 3 (left) shows the measured strains of the FBGs as a function of the drill hole depth. The nine measurement sections of the FBG sensor used have a grating length of $8 \mathrm{~mm}$. As can be seen, these sensors are not able to measure the strains during the entire drilling process. The FBGs which are positioned under the first plane at a depth of $3 \mathrm{~mm}$ only measure until the hole is drilled out to a depth of $3 \mathrm{~mm}$. Accordingly, the sensors under the second plane show this limiting behaviour at $6 \mathrm{~mm}$ drill hole depth. The strain evaluation with the sensors at the bottom of the sample fails at a drill hole depth of $7 \mathrm{~mm}$. The cause of this effect is the inhomogeneous strain field created by the drill hole. The Bragg grating is affected by a high strain gradient due to the alternation of tensile and compressive strains, as shown in Figure 3 (right). In addition, a load occurs on the fibre in transverse direction. This results in a widening and even splitting of the reflection peak, which means an evaluation based on homogeneous strains is no longer possible. These results were shown in [8].

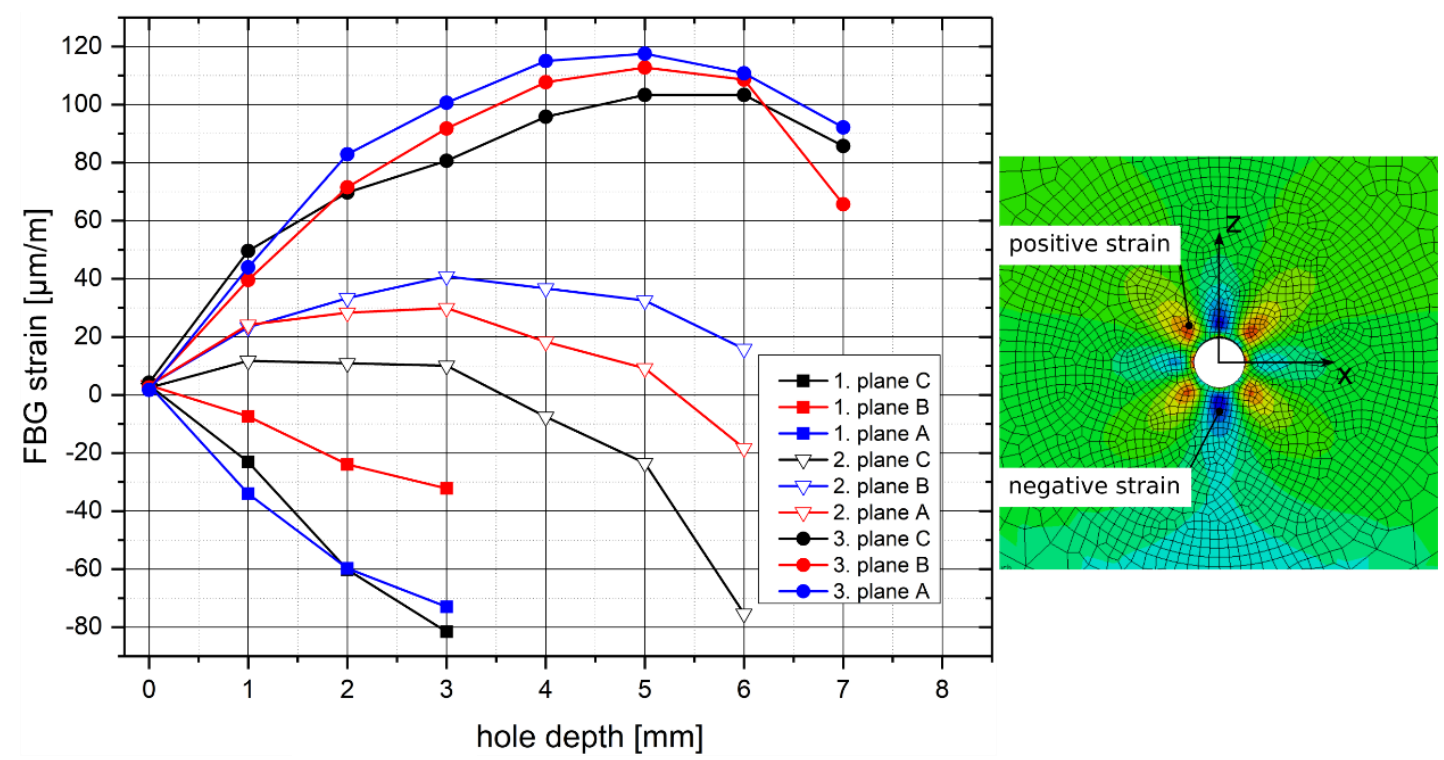

Figure 3: Strains of the FBG sensors with a Bragg grating length of $8 \mathrm{~mm}$ (left); qualitative numerical strain distribution in z-direction around the drill hole (right) 
The effect described above can be reduced by shortening the Bragg gratings. For this investigation a sample with Bragg grating of $3 \mathrm{~mm}$ length was manufactured. The strain results are depicted in Figure 4.

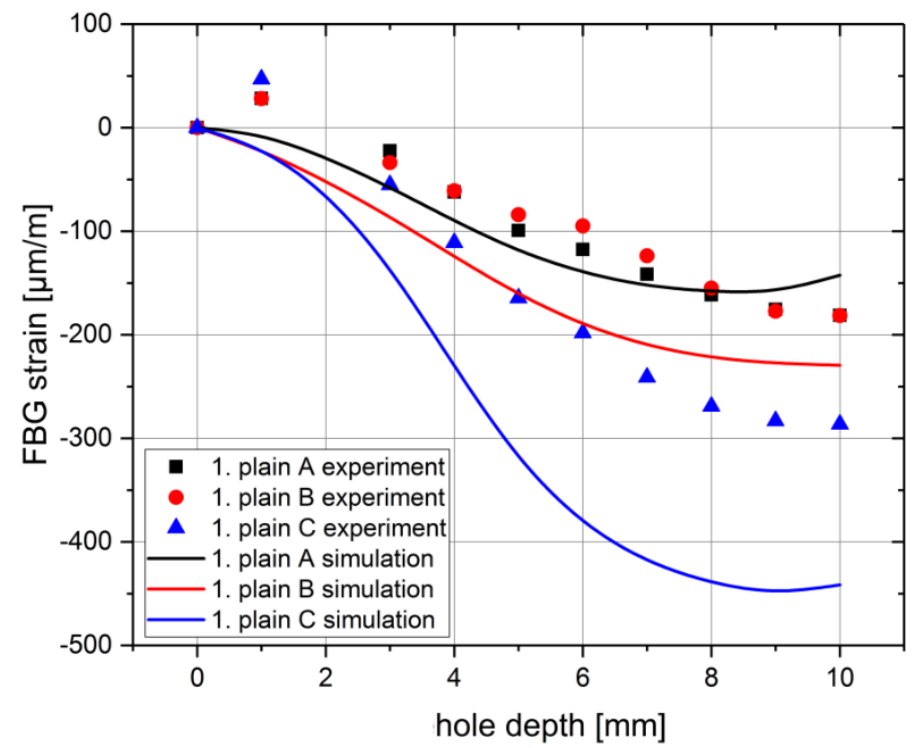

Figure 4: Strains of the FBGs with a Bragg grating length of $3 \mathrm{~mm}$ and continuously simulated strains

The FBGs with the short Bragg lengths are suitable for monitoring the whole drilling process. The strain values are smooth and show a decreasing trend. In addition to the practical execution, the experiment was also modelled by numerical simulations with the FE software ABAQUS. For the simulation, the epoxy material is assumed as an elastic material with a Young's modulus of $3000 \mathrm{MPa}$ and Poisson's ratio of 0.3 . The calculated strains are also shown in Figure 4. The distributions of experiment and simulation correspond qualitatively, but the quantitative agreement is still insufficient. Therefore a fundamental investigation of the transmission behaviour and the behaviour of the Bragg grating with inhomogeneous deformations was conducted, as describe in the following section.

\section{Investigation of FBG sensors under inhomogeneous deformations}

\subsection{Sample development}

The inhomogeneous deformations, as shown in section 2, result in problems for when evaluating FBG measurements. This phenomenon causes a splitting of the reflected wavelength peak. The standard strain evaluation theory provides no answer as to how the split peak should be handled and how the deformation is calculated. Thus, an experiment was carried out using FBG sensors in a section within inhomogeneous deformations. Several possibilities exist to generate inhomogeneous deformations (e.g. introduction of notches, discontinuous loads, or geometries). In [9], a tensile test setup with discontinuous geometry is described. The experimental setup, which is used in this section, includes notched aluminium beams, which are used for a four-point bending test. The sensors are applied on the opposite (planar) side of the notch.

The notches were chosen by an iterative numerical optimisation. Thereby three different notch geometries were identified, produced and characterised as shown in Figure 5. For the comparison, an unnotched beam was investigated, too. 


\begin{tabular}{|c|c|c|c|c|}
\hline \multirow{2}{*}{ case } & $\mathrm{R}$ & $\mathrm{a}$ & $\mathrm{t}$ \\
\cline { 2 - 5 } & \multicolumn{3}{|c|}{ in $\mathrm{mm}$} \\
\hline 1 & & 0 & 0 & 0 \\
\hline 2 & $\cdots \cdots \cdots$ & 10 & 3 & 10 \\
\hline 3 & -- & 10 & 0 & 10 \\
\hline 4 & $-\cdots-$ & 5 & 0 & 15 \\
\hline
\end{tabular}

1

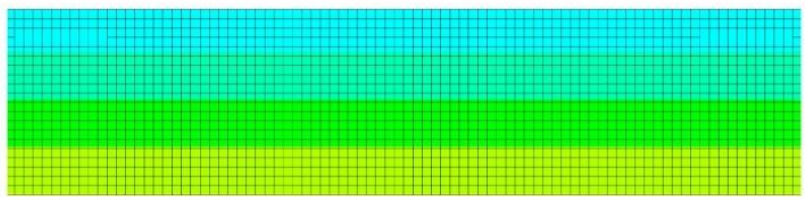

2
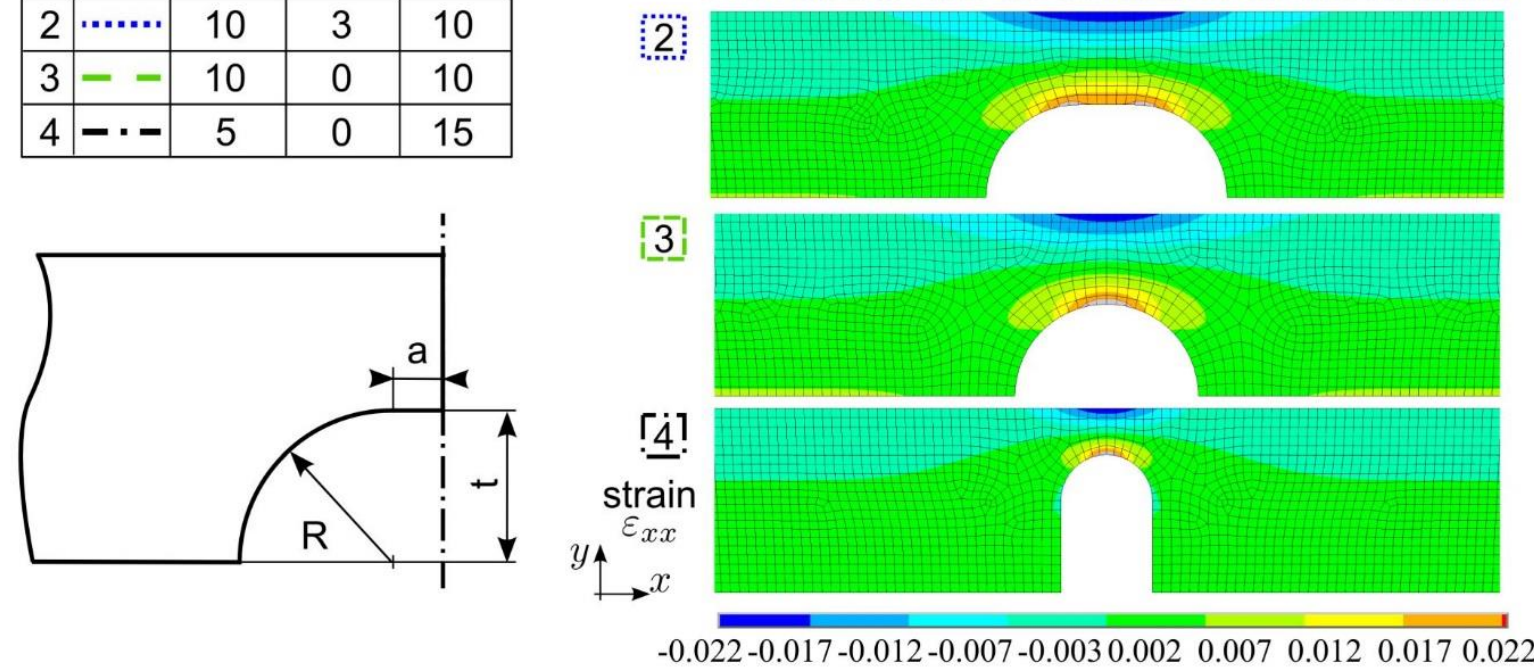

Figure 5: Characterisation of the three notched geometries used and numerical results of the bending load

Figure 6 shows a distribution of the normalised strain value (actual strain divided by the maximum strain along a path) as a measure of the strain gradient.

Different sensors are applied to each beam, as shown in Figure 7. There is one strain gauge in the centre above the notch. Next to this strain gauge two FBG sensors are applied. One is aligned with its edge in the centre of the notch (eccentric sensor), the other one is placed centrally above the notch (centric sensor). The strain gauge is used as reference, whose strain is the constant value of each experiment.
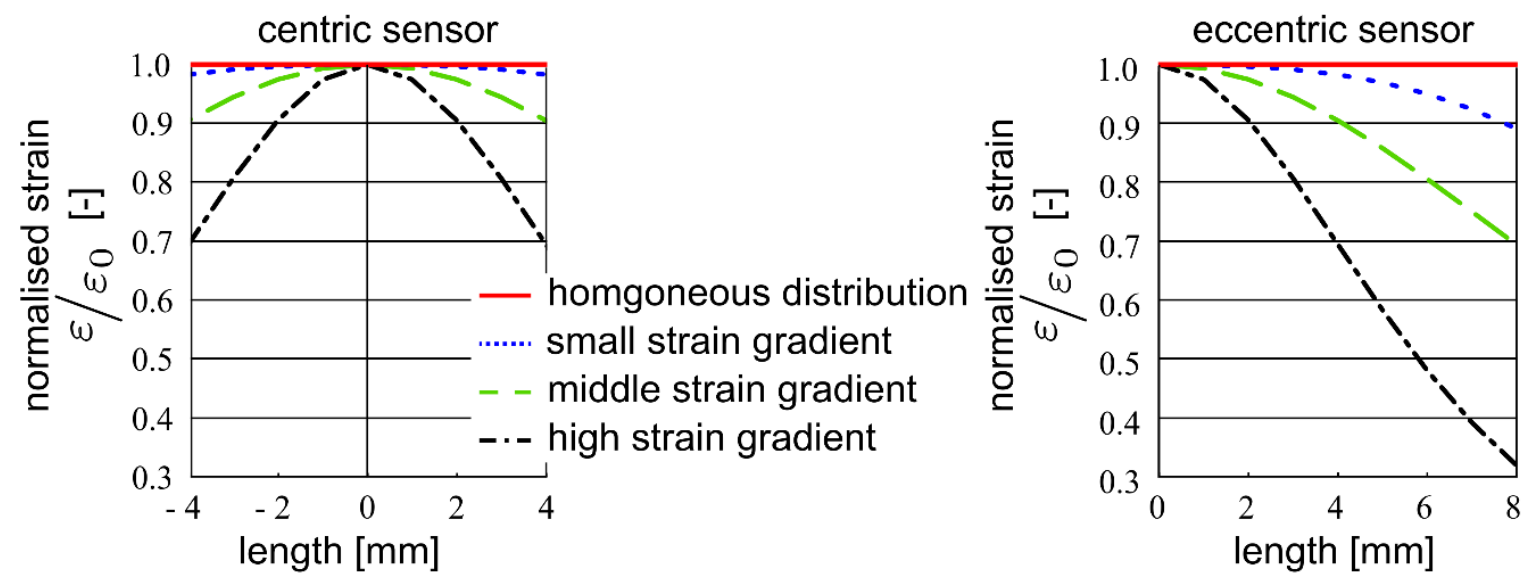

Figure 6: Distribution of the strain gradient of the centric and eccentric FBG sensor

The experimental setup includes a four-point bending test, which is installed in a test machine (see Figure 7). First, the beam is loaded with a small preload of $3 \mathrm{~N}$. Subsequently, a defined displacement is applied. The applied displacements depend on the beam and are adjusted in such a way that the resulting strain gauge strain is $2000 \mu \mathrm{m} / \mathrm{m}$. When the maximum displacement is reached, the beam is unloaded. The whole experiment is repeated three times. In additional experiments the beam is turned so that the sensor side is stressed with tension and compression. 


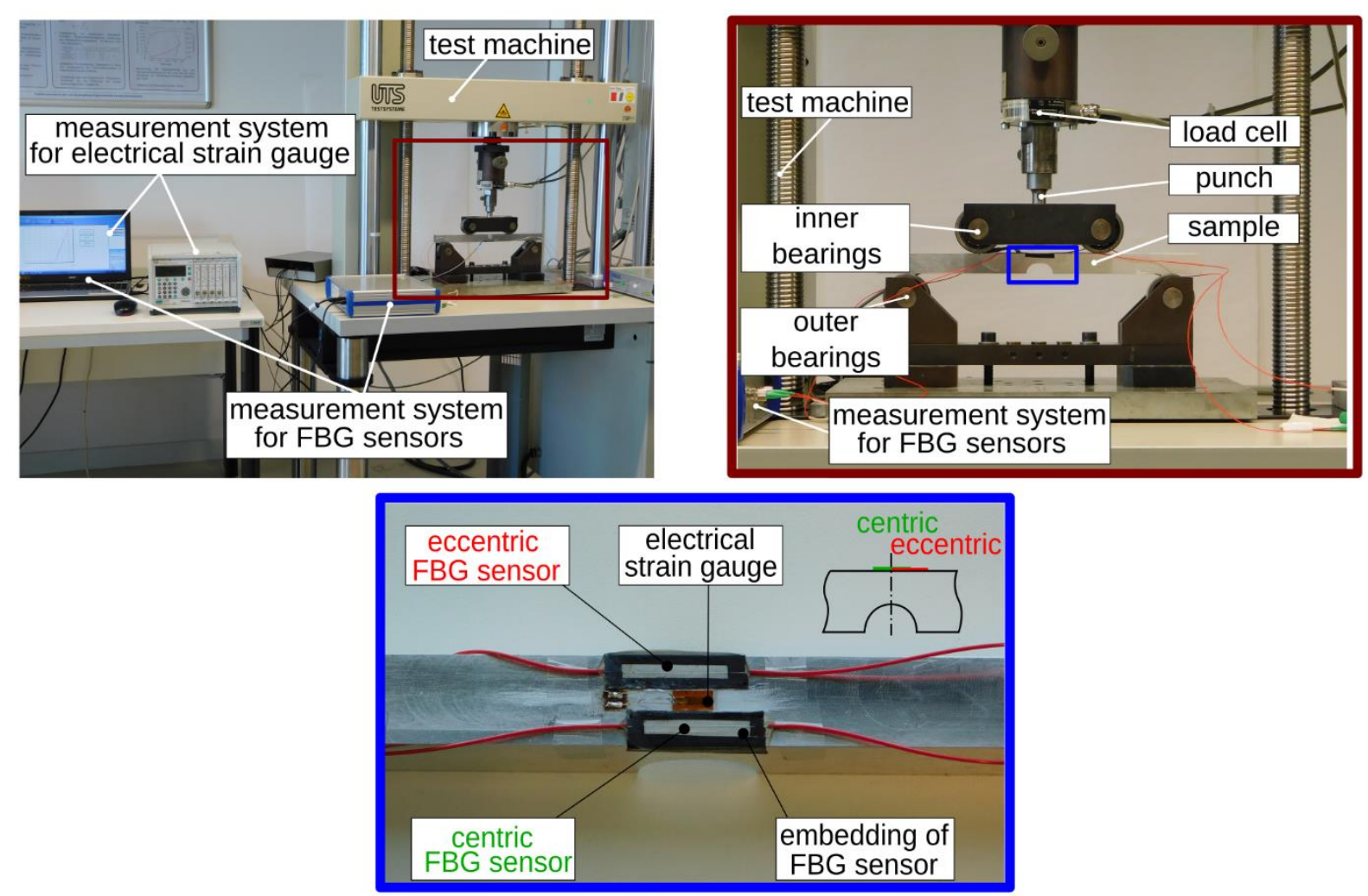

Figure 7: Experimental setup of four-point bending test and sensor application of strain gauge and two FBG sensors

\subsection{Results}

In the following, the results of the four-point bending experiment are presented and discussed. First, the strain distribution of the FBG sensors over time and the strain gauge of the notched beam with low strain gradient are shown in Figure 8 (left). It is noticeable that the agreement of the strain values is excellent. This can also be confirmed by Table 1, where the force and strain values for the three repetitions are listed. Similar results for the repetition accuracy of the measured force could also be achieved for the beams with different notches. Figure 8 (right) shows the FBG signal intensities at different longitudinal strains as a function of the wavelength shifts of the centric and eccentric sensors. No significant change of the narrowband peak is recognisable, so that the fundamental equations for standard evaluation can be applied.
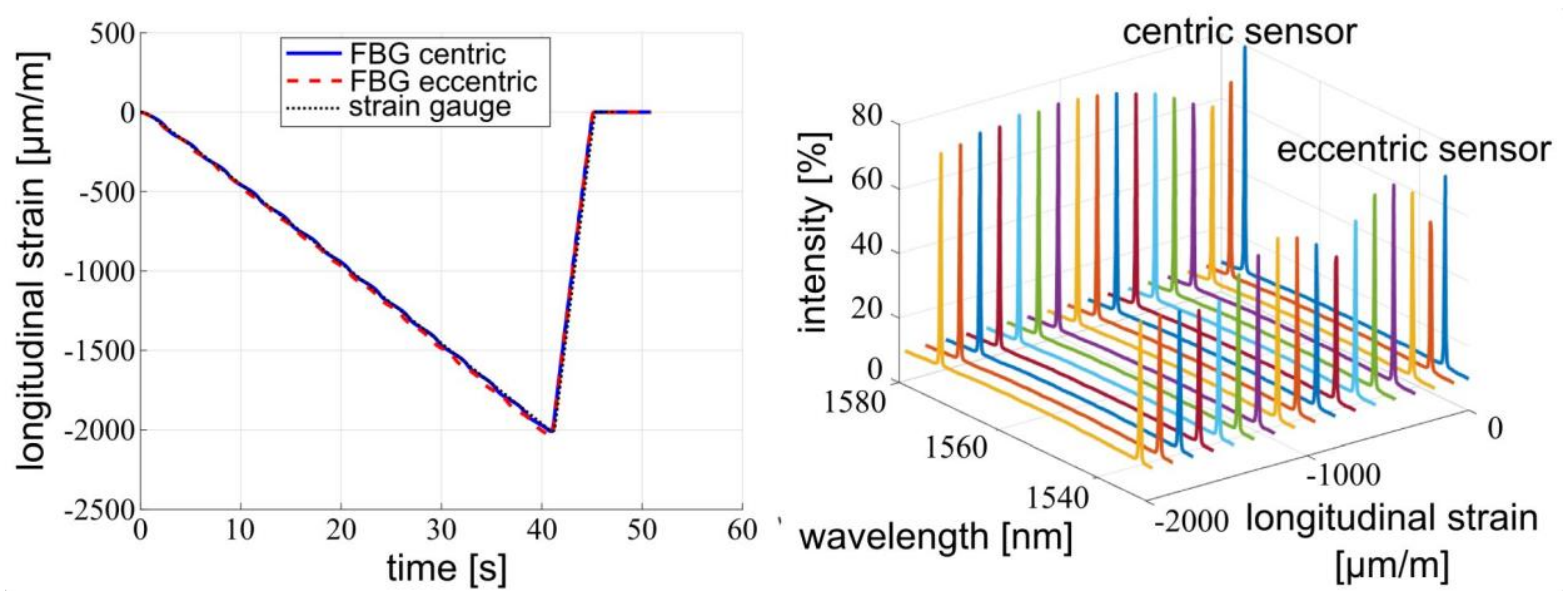

Figure 8: Strain distribution over time (left) and intensity as a function of wavelength shift and strain (right) for the small strain gradient beam under compression 
Table 1: Summary of the repetition of the experiments with the notched beam with small strain gradient under compression

\begin{tabular}{lllll}
\hline Sample & $\boldsymbol{F}_{\max }[\mathbf{N}]$ & $\boldsymbol{\varepsilon}_{\text {strain gauge }}[\boldsymbol{\mu m} / \mathbf{m}]$ & $\boldsymbol{\varepsilon}_{\mathrm{FBG}, \text { centric }}[\boldsymbol{\mu m} / \mathbf{m}]$ & $\boldsymbol{\varepsilon}_{\mathrm{FBG}, \mathrm{eccentric}}[\boldsymbol{\mu m} / \mathbf{m}]$ \\
\hline 1 & 1435 & -2011 & -2009 & -2038 \\
2 & 1437 & -2014 & -2014 & -2040 \\
3 & 1439 & -2016 & -2017 & -2042 \\
\hline average & 1437 & -2013 & -2013 & -2040 \\
standard deviation & 2 & 2 & 3 & 2 \\
\hline
\end{tabular}

Next, the results of the notched beam with medium strain gradient are shown in Figure 9 (left). Similar to the previous results with small strain gradients the strain analysis can be used. However, it can be seen that the longitudinal strain of the centric sensor differs from the others. This signal is already affected by peak widening.
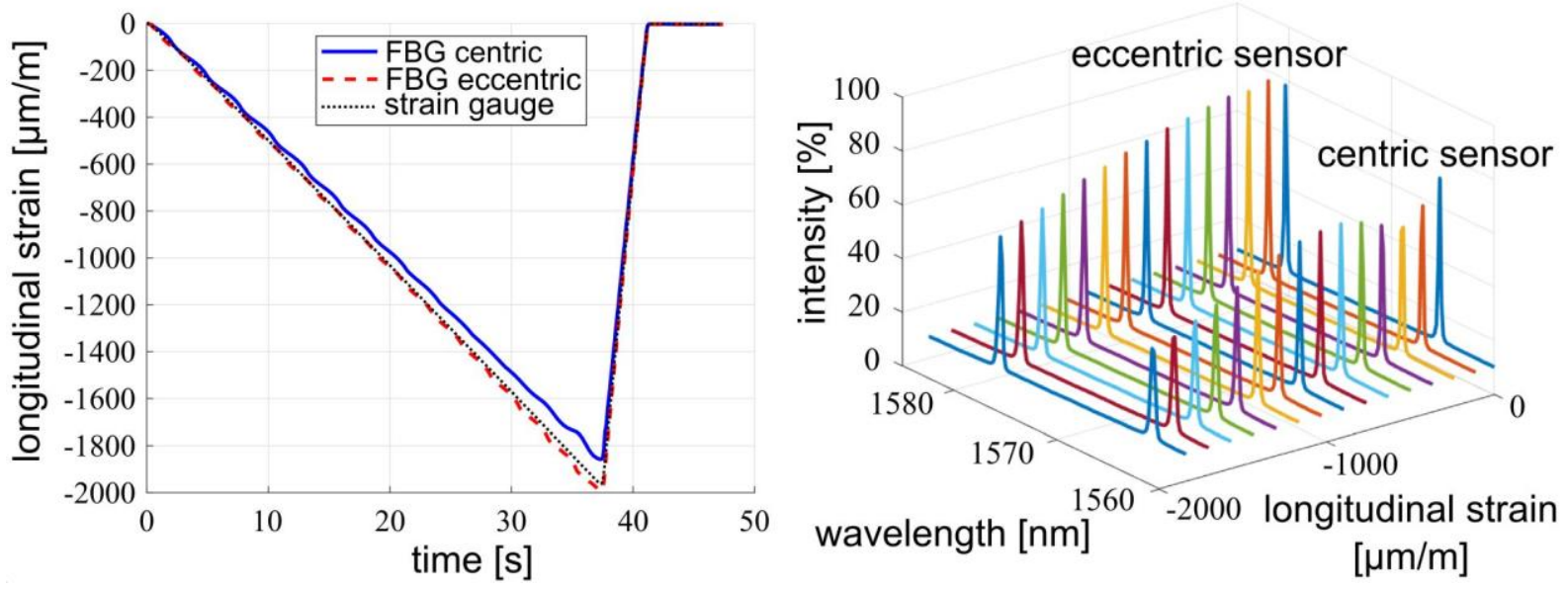

Figure 9: Strain distribution over time (left) and intensity as a function of wavelength shift and strain (right) for the medium strain gradient beam under compression

The most significant results are the wavelength shifts of the beam with the high strain gradient, which are shown in Figure 10. In this case the sensor's intensity already decreases and starts to widen asymmetrically. This asymmetrical widening results in the peak splitting. Here, a splitting of the reflected peak can be seen for the eccentric sensor at longitudinal strain values of approximately $-1190 \mu \mathrm{m} / \mathrm{m}$. The centric sensor splits at approximately $-1220 \mu \mathrm{m} / \mathrm{m}$. This demonstrates that the sensor with the higher strain gradient is more sensitive to peak degeneration. Before splitting of the peaks a widening occurs and the intensity decreases. If a peak widens, then the standard analysis methods for the evaluation of the Bragg wavelength $\lambda_{B}$ are debatable. If the signal splits, then these methods are completely inapplicable. 


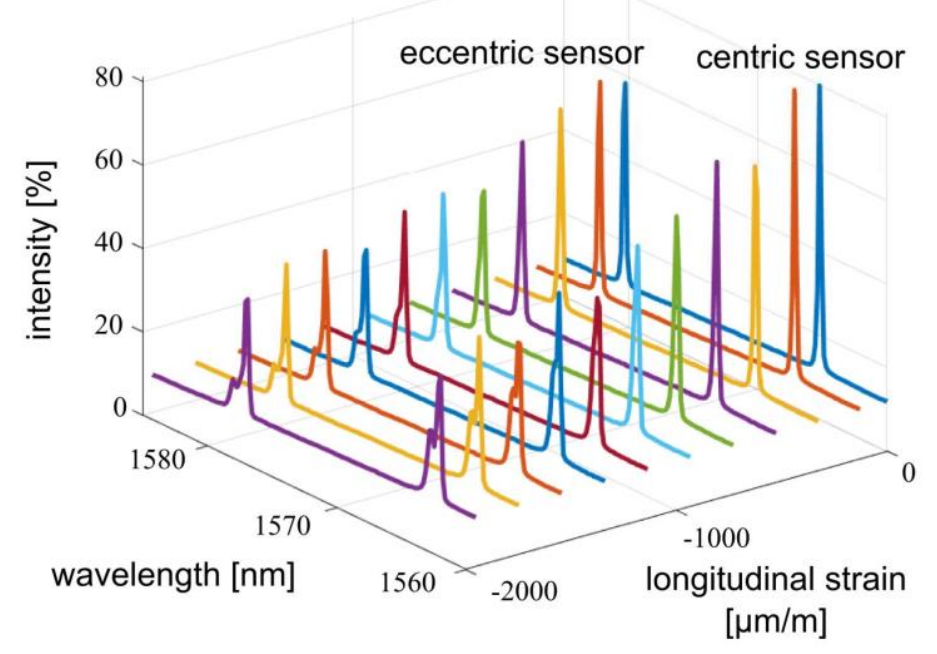

Figure 10:Structure Intensity as a function of wavelength shift and strain for the high strain gradient beam under compression

\section{Conclusion and outlook}

First, an experimental setup was introduced in which residual stresses were analysed by FBG sensors demonstrating the challenges of strain evaluation under inhomogeneous deformation conditions. In addition, a four-point bending test was introduced for three different notched aluminium beams and the effects on the FBGs were investigated. The results show that the experiment is reproducible and delivers valid results. Furthermore, it can be seen that the peak widening has an impact on the strain analysis and that inhomogeneous deformations cause peak splitting resulting in an inaccurate strain calculation.

To solve these challenges, the approach to be pursued is to reproduce the reflected spectra with mathematical and optical models. The expectation is that more mechanical information can be extracted from the reflected spectra for the calculation of strain states around fibre optic sensors.

\section{Acknowledgements}

This work was performed within the Federal Cluster of Excellence EXC 1075 "MERGE Technologies for Multifunctional Lightweight Structures" and supported by the German Research Foundation (Deutsche Forschungsgemeinschaft, DFG). It is also part of a measure which is co-financed by tax revenue based on the budget approved by the members of the Saxon state parliament. The financial support is gratefully acknowledged.

\section{References}

[1] Ospald, F.; Herzog, R.: Topology Optimization for Injection Molding of Short Fiber-Reinforced Plastics. In: J Proc. Appl. Math. Mech. 17, 2018, pp. 337-338. doi:10.1002/pamm.201710138

[2] Goldberg, N. et al: Considering the fiber orientation for the computation of the hyperelastic Tucker average for short fiber reinforced composites. 29th Workshop Composite Forschung in der Mechanik 6. -8.12.2016, Bad Herrenalb, 2016, p. 10.

[3] Hannusch, S. et al: Efficient Simulation, Optimization, and Validation of Lightweight Structures. In: IMTC 2015 Lightweight Structures Chemnitz, 2015, pp. 219-227.

[4] ASTM E837-08: Standard test method for determining residual stresses by the hole-drilling strain-gauge method, 2008. www.astm.org.

[5] Luyckx, G. et al: Strain Measurements of Composite Laminates with Embedded Fibre Bragg Gratings: Criticism and Opportunities for Research. In: Sensors 11 (2011) 1, pp. 384-408. 
doi: $10.3390 / s 110100384$

[6] Kersey, A. D. et al: Fiber grating sensors. In: J. Lightwave Technol. 15 (1997) 8, pp. 1442-1463. doi: $10.1109 / 50.618377$

[7] Balek, V. et al: A new approach to characterization of barrier properties of ORMOCER protective coatings, In: J of Sol-Gel Science and Technology 8) (1997), pp. 591-594. doi: 10.1007/BF02436905

[8] Peretzki, E. et al: Residual stress analysis of fibre-reinforced structures with fibre BRAGG grating sensors. In: IMTC 2017 Lightweight Structures Chemnitz, 2017, pp. 99-100.

[9] Peters, K. et al: Experimental verification of response of embedded optical fiber Bragg grating sensors in non-homogeneous strain fields. In: J Optics and Lasers in Engineering, 2000, pp. 107-119. 